\title{
3,3'-Diindolylmethane induces miRNA-132 to potentiate cholinergic anti- inflammation and suppress autoimmune encephalomyelitis
}

\author{
Hanieh Hamza \\ Biological Sciences Department, King Faisal University, Ahsaa 31982, Saudi Arabia \\ Corresponding author e-mail: hhanieh@kfu.edu.sa
}

\begin{abstract}
The microRNAs (miRNAs) are a group of non-coding RNAs that regulate gene expression at the post-transcriptional level. An increasing paradigm has shown that these molecules are critically implicated in pathogenesis of multiple sclerosis (MS). However, the axis of miRNAs with the proteins that show important roles in MS is largely anonymous. Previously, our data showed that activation of the transcription factor aryl hydrocarbon receptor (Ahr) by 2, 3, 7, 8tetrachlorodibenzo-p-dioxin (TCDD) alleviates experimental autoimmune encephalomyelitis (EAE), a mouse model of MS. This effect was attributed to up-regulation of miR-132 that targets acetylcholinesterase (AChE). The 3, 3'-diindolylmethane (DIM)-activated Ahr also alleviates inflammatory symptoms of EAE, but no studies have linked DIM with miR-132. Data of the current study showed that miR-132 was down-regulated in encephalitogenic CD4 ${ }^{+}$cells, while DIM restored the miR-132 level. The DIM treatment up-regulated AChE-targeting miR-132, which in turns potentiated cholinergic anti-inflammation in EAE mice. Transfection of CD4 ${ }^{+}$ cells with miR-132 mimics decreased cell proliferation, interleukin (IL)-17, interferon (IFN)- $\gamma$ and enhanced production of the transforming growth factor (TGF)- $\beta$. In conclusion, the findings identify a new miRNA-based mechanistic explanation for the anti-inflammatory properties of DIM in EAE, suggesting a promising therapeutic potential of miR-132 to control autoimmune inflammation in MS patients.
\end{abstract}

Key words: 3, 3'-diindolylmethane, experimental autoimmune encephalomyelitis, miR-132, cholinergic anti-inflammation

\section{INTRODUCTION}

The aryl hydrocarbon receptor (Ahr) is a heterodimeric transcription factor that binds to a large number of exogenous agonists such as 3,3'-diindolylmethane (DIM), 2,3,7,8-tetrachlorodibenzo-p-dioxin (TCDD) and 3-methylcholanthrene (3MC) (Hanieh 2014). For decades, the Ahr has been known to mediate the mechanisms that underlie environmental toxicity and immunotoxicity. However, later progress has expanded the functions of Ahr in the immune system to include aspects of antibody production, inflammation and autoimmunity (Nguyen et al 2013). Therefore, understanding the molecular mechanisms that underlie the Ahr actions represents an intriguing strategy for treatment of immune disorders.

In previous studies, we and others have shown that Ahr is critically involved in the development of different autoimmune disease (Quintana et al 2008; Stockinger et al 2009; Nakahama et al 2011; Nakahama et al 2013; Chinen et al 2015), and secretion of pro-inflammatory cytokines (Masuda et al 2011; Nguyen et al 2013). Accumulating evidences have shown that activation of Ahr by certain exogenous agonists attenuates autoimmune inflammation. For instance, DIM and TCDD alleviate inflammation in different animal models of autoimmune diseases including experimental autoimmune 
encephalomyelitis (EAE) (Quintana et al 2008; Rouse et al 2013), ulcerative colitis (UC) (Singh et al 2011), and rheumatoid arthritis (RA) (Dong et al 2010). However, the molecular mechanisms that underlie the therapeutic properties of Ahr agonists remain to be investigated.

The microRNAs (miRNAs) are a group of non-coding RNAs ( 20nt) that regulate gene expression by complementary binding on the 3' untranslated region (3'UTR) of the target mRNA(s). The complementary binding regulates gene expression at the posttranscriptional level by degradation of mRNA or blockade of protein translation. Numerous studies have demonstrated that miRNA are implicated in different functions of the immune system in health and disease such as lymphocytes development, normal immune responses and development of autoimmunity (de Yébenes et al 2013; Liu and Abraham 2013; Zhu et al 2013).

Of special interest, it has been demonstrated that miRNAs modulate autoimmune inflammation at different levels. Recently formed paradigm has clearly unraveled the physiological significance of several miRNAs in animal models of multiple sclerosis (MS). The let-7e, miR-21, miR-155 and miR-326 are highly expressed in EAE mice (Du et al 2009; O'Connell et al 2010; Guam et al 2013; Murugaiyan et al 2015). Knockout or knockdown of these miRNAs decreases the inflammatory $\mathrm{T}$ cells and attenuates disease symptoms. Although different roles of miRNAs and $\mathrm{Ahr}$ in autoimmune inflammation are currently demonstrated, their axis is rarely investigated.

The miR-132 is a member of a highly conserved miRNA cluster located in chromosome 17 in humans and chromosome 11 in micenamed miR-212/132. Previous studies have linked miR-132 expression to autoimmune inflammation. For example, a differential expression of miR-132 is detected in synovial fluid and joint tissues of RA patients (Pauley et al 2008; Murata et al 2010; Filková et al 2014), and in $\mathrm{CD}^{+}$cell from EAE mice (Hanieh and Alzahrani 2013). It has been also shown that miR-132 resolves inflammation by functional regulation of the brain-to-body communication (Shaked et al 2009). It potentiates cholinergic anti-inflammation by targeting the pro-inflammatory mediator acetylcholinesterase (AChE), an enzyme hydrolyzes the acetylcholine (ACh) and mitigates its anti-inflammatory functions. In our hands, we showed that activation of Ahr by the synthetic toxic agonist TCDD attenuates EAE by inducing AChE-targeting miR-132 (Hanieh and ALzahrani 2013). Therefore, current work hypothesized that activation of Ahr by the natural agonist DIM would ameliorate autoimmune inflammation in EAE model by inducing the miR132/AChE module.

To test the hypothesis, the effect of DIM on miR-132 expression and miR132/AChE module in EAE mice has been studied. The results, for the first time, show that activation of Ahr by DIM induces miR132 expression and potentiates cholinergic anti-inflammation in vitro and in vivo. The induced anti-inflammatory system attenuates autoimmune inflammation in EAE mice by suppression of pro-inflammatory mediators and proliferation of encephalitogenic $\mathrm{CD} 4^{+}$ cells.

\section{MATERIALS and METHODS}

\section{Mice}

Female, 6-8 weeks old C57BL/6 mice were purchased from King Faisal Specialist Hospital and Research Center, Riyadh, KSA. All animals were maintained under specific-pathogen-free (SPF) conditions, and had free access to food and water. Animal experiments were performed in accordance with the regulations of the 
Animal Care Committee of King Faisal University, Hofuf, KSA.

\section{EAE model and DIM treatment}

The EAE was induced as described previously (Hanieh and Alzahrani 2013). Mice were injected subcutaneously at the base of the tail with $100 \mu \mathrm{g} / \mathrm{mL}$ of $\mathrm{MOG}_{35-55}$ (Peptides International, Louisville, KY) emulsified in complete Ferund's adjuvant (CFA) containing $5 \mathrm{mg} / \mathrm{mL}$ of $M$. tuberculosis H37Ra (Difco Laboratories, Detroit, MI). The mice were injected intraperitoneally with $225 \mathrm{ng}$ of pertussis toxin (Sigma Aldrich, St Louis, MO) on days 0 (MOG $35-55^{-}$immunization day) and 2 days later.

Animals were gavaged with DIM (60 $\mathrm{mg} / \mathrm{kg} /$ day) or vehicle alone (corn oil) for 7 consecutive days starting one day prior to EAE induction. The EAE symptoms were assessed using the following score: 0 , no clinical symptoms; 1 , limp tail; 2 , hind limb paresis; 3, forelimb weakness; 4, paralysis; 5 , death. Naïve mice, the vehicle-treated, were used as negative control. As sampling days indicated, mice were euthanized on day $7,10,18$ or 25 after $\mathrm{MOG}_{35-55^{-}}$ immunization

\section{Real-time PCR (qPCR)}

Total RNA was extracted using RNeasy RNA extraction kit (Qiagen, Valencia, CA) then reverse transcribed in a thermal cycler using reverse transcriptase. The TaqMan Gene expression assays of miR-132 and its endogenous control RNU6, and AChE and its endogenous control GAPDH were used. The ViiA7 system was used for qPCR with cycling conditions $50^{\circ} \mathrm{C}$ for $2 \mathrm{~min}$ and $95^{\circ} \mathrm{C}$ for $10 \mathrm{~min}$, then 40 cycles of $95^{\circ} \mathrm{C}$ for $15 \mathrm{sec}$ and $60^{\circ} \mathrm{C}$ for 1 min. System and reagents for qPCR were all from Applied Biosystems, Grand Island, NY. The $\Delta \Delta C t$ method was used for calculations of the expression fold change.

\section{Protein measurements}

Serum IL- $1 \beta$, IL-6 and TNF- $\alpha$, and supernatant IL-17, IFN- $\gamma$, TGF- $\beta$ and IL-10 were measured using Quantikine ELISA kits according to the manufacturer's instructions (R\&D systems, Minneapolis, MN). The splenocytes were lysed using RIPA lysis buffer system (Santa Cruz Biotechnology, Santa Cruz, CA). The lysate was then fractionated on SDS-page, and AChE and $\beta$ actin were detected using their specific antibodies (Santa Cruz Biotechnology).

\section{Cell culture}

The $\mathrm{CD}^{+}$cells from spleens of EAE mice were isolated using CD4 isolation kit (MACS; Miltenyi Biotec, Auburn, CA), and then restimulated with $\mathrm{MOG}_{35-55}$ (25 $\mu \mathrm{g} / \mathrm{mL})$. Activation of Ahr in vitro was induced by DIM at $30 \mu \mathrm{mol} / \mathrm{L}$. The cells were cultured in complete RPMI-1640 medium supplemented with $10 \%$ fetal bovine serum (FBS) and penicillin/streptomycin (Sigma Aldrich). The cells were incubated at $37^{\circ} \mathrm{C}$ and $5 \%$ $\mathrm{CO}_{2}$ in a humidified atmosphere.

\section{Transfection}

MiR-132 antisense, miR-132 mimics and negative controls (Ambion, Austin, TX) were transfected into spleen $\mathrm{CD}^{+}$cells by 4D-nucleofector device and $\mathrm{T}$ Cell Nucleofector Kit following manufacturer's instructions (Lonza, Walkersville, MD). The efficiency of the transfection was confirmed by qPCR.

\section{Catalytic activity of AChE}

The catalytic activity of $\mathrm{AChE}$ in the supernatant of $\mathrm{CD}^{+}$cells culture was measured using modified Ellman's method (Ellman et al 1961). The $\mathrm{CD}^{+}$cells from naïve mice were stimulated with PHA (7 $\mu \mathrm{g} / \mathrm{mL}$; Sigma Aldrich). The ACh decay was quantified at 12, 24 and $36 \mathrm{~h}$ with the existence of butyrylcholinesterase (BChE) inhibitor (Sigma Aldrich). 


\section{T cell Proliferative response}

The spleen $\mathrm{CD}^{+}$cells from $\mathrm{EAE}$ mice were seeded in 96-well flat-bottom plates in triplicates and restimulated with $\mathrm{MOG}_{35-55}$ for 2 days. Cell proliferation was quantified by MTT assay using CCK-8 kit (Dojindo, Baltimor, MD), following manufacturer's instructions.

\section{Statistical Analysis}

The scores of EAE over the experimental period were analyzed by Analysis of variance (ANOVA) two-factor test. Other parameters were tested using ANOVA single factor test. $\mathrm{P}<0.05$ was considered significant. Data are presented as mean \pm SD of pooled data from three independent experiments.

\section{RESULTS}

\section{Oral DIM treatment alleviates EAE in vivo}

The effects of DIM on autoimmune inflammation were examined in mouse model of MS. The EAE model was induced in mice using myelin oligodendrocyte glycoprotein $\left(\mathrm{MOG}_{35-55}\right)$ in complete Freund's adjuvant (CFA), and pertussis toxin. The oral treatment of DIM, (60 $\mathrm{mg} / \mathrm{kg} /$ day) for 7 days starting one day prior to EAE induction, obviously decreased EAE scores and delayed disease onset by $2.4 \pm$ 0.6 day compared with control mice (Fig. 1A). The mean of maximum score of EAE were lower in DIM-treated mice compared with control mice (Fig. 1B). Serum collected from EAE mice at the peak of disease (18 days) showed that the suppressive effects of DIM on disease score were concomitant with lower levels of serum IL-1 $\beta$, IL- 6 and TNF- $\alpha$ (Fig. 1C).

\section{DIM induces miR-132 in spleen $\mathrm{CD}^{+}$in vivo}

To examine whether miR-132 was involved in EAE development at early stages, miRNA expression was quantified in spleen encephalitogenic $\mathrm{CD}^{+}$cells. Induction of EAE significantly downregulated miR-132 expression in spleen $\mathrm{CD}^{+}$cells 7 days after $\mathrm{MOG}_{35-55}$ immunization compared to the naïve healthy mice (Fig. 1D). To further examine the involvement of miR-132 in EAE development, the miRNA expression was quantified at later stages, day 20 after $\mathrm{MOG}_{35-55}$ immunization. As shown in Fig. 1E, miR-132 expression profile in $\mathrm{CD}^{+}$ cells from EAE was comparable to that quantified on the $7^{\text {th }}$ day after immunization. Interestingly, oral DIM treatment restored miR-132 expression in $\mathrm{CD}^{+}$at early and late EAE development stages to a level comparable to that of the naïve mice. Taken together, the findings showed for the first time that DIM induced miR-132 in spleen $\mathrm{CD}^{+}$in vivo under autoimmune conditions and that DIM-induced miR-132 could be involved in the anti-inflammatory effects of DIM. 

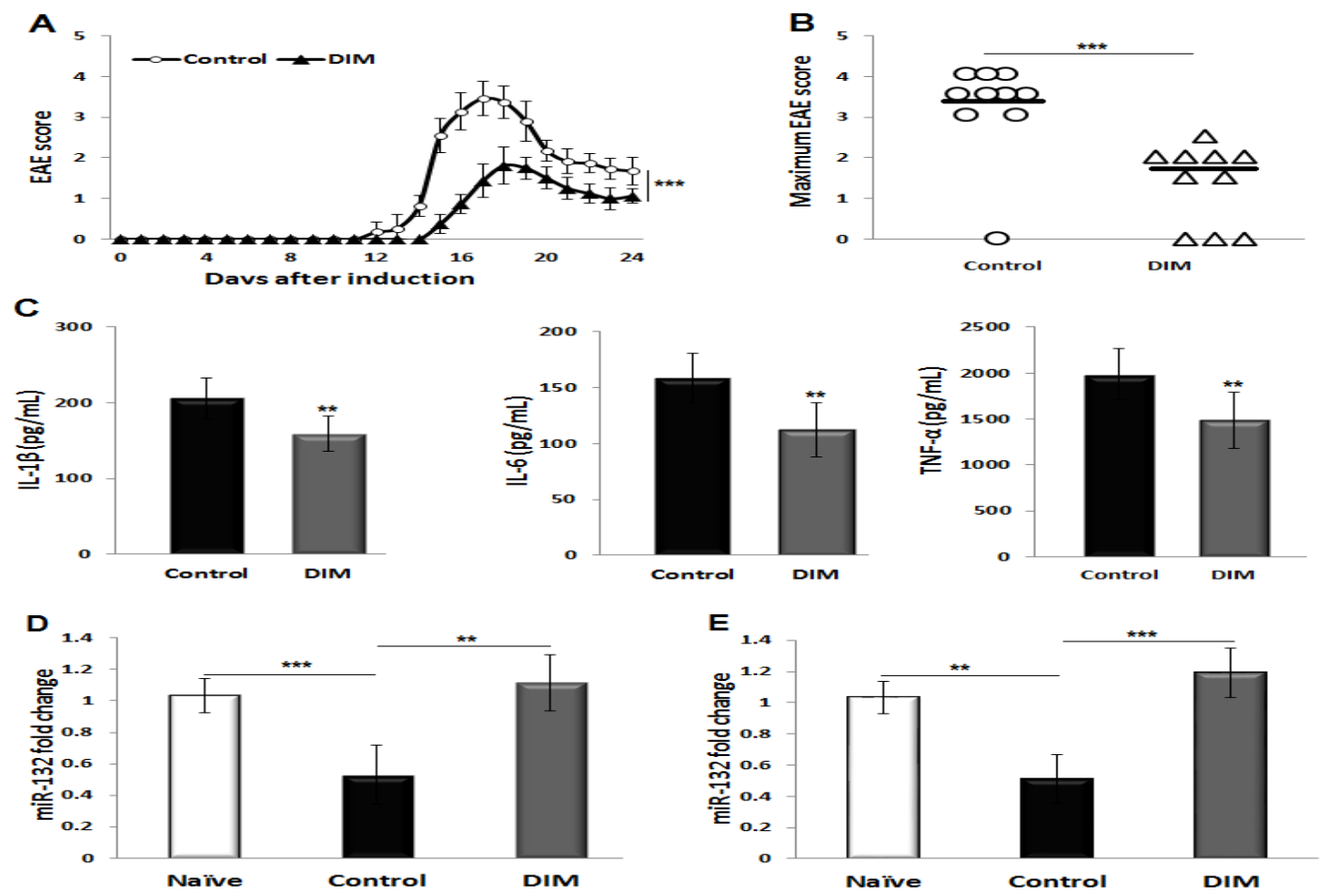

Figure 1. Oral DIM treatment alleviates EAE and up-regulates miR-132 expression in vivo. (A) Mean EAE clinical score. (B) Maximum clinical score distribution. The EAE mice were gavaged with DIM $(60 \mathrm{mg} / \mathrm{kg} / \mathrm{day})$ or vehicle (corn oil) for 7 days starting one day prior to the immunization day. (C) Serum level of IL-1 $\beta$, IL-6 and TNF- $\alpha$ in EAE mice 18 days after $\mathrm{MOG}_{35-55}$ immunization. MiR-132 expression in spleen $\mathrm{CD}^{+}$cells 7 days (D) and 20 days (E) after immunization; Naïve: negative control. $* * p<0.01, * * * p<0.001$. Data are shown as mean \pm SD of pooled data from three independent experiments. (A) Control $n=11$ and DIM $n=8,(B) n=10$, (C) $n=8$ and (D) $n=5$.

\section{DIM potentiates cholinergic anti- inflammation by inducing miR-132/AChE module}

It is reported that miR-132 targets the mRNA of the pro-inflammatory mediator AChE (Shaked et al 2009). Also, TCDD induces the miR-132/AChE module in EAE mice 9 days after $\mathrm{MOG}_{35-55}$ immunization (Hanieh and Alzahrani 2013). Therefore, the effect of DIM on AChE gene expression in spleens of EAE mice was studied 10 days after immunization. As shown in Fig. 2A and $\mathrm{B}$, the DIM reduced $\mathrm{AChE}$ gene expression 10 days after immunization compared with the control EAE mice. These results suggest that DIM, similar to TCDD, is implicated in a cholinergic antiinflammation in EAE mice.
To further investigate whether DIM induced cholinergic anti-inflammation comparable to that obtained by TCDD, the effect of DIM on the catalytic activity of $\mathrm{AChE}$ in $\mathrm{CD}^{+}$ cells was studied. Results showed that DIM suppressed phytohaemagglutinin (PHA)induced $\mathrm{AChE}$ catalytic activity on $\mathrm{ACh}$ (Fig. 2C). These results suggest that DIM induces cholinergic anti-inflammation in $\mathrm{CD}^{+}$cells. To investigate whether the DIMsuppressed AChE was mediated by miR132, a direct effect of miR-132 was examined. Over-expression of miR-132 by miRNA mimics (mim-miR-132) significantly suppressed the catalytic activity of $\mathrm{AChE}$ to a level that was significantly lower than that of DIM (Fig. 2C). Next, to analyze whether the effect of DIM on AChE catalytic activity was mediated by miR-132, 
the miRNA was inhibited by antisense (as$\mathrm{miR}$ ) in the DIM-treated $\mathrm{CD}^{+}$cells. Transfection of as-miR-132 partially abrogated the DIM-suppressed AChE catalytic activity (Fig. 2D). Taken together,
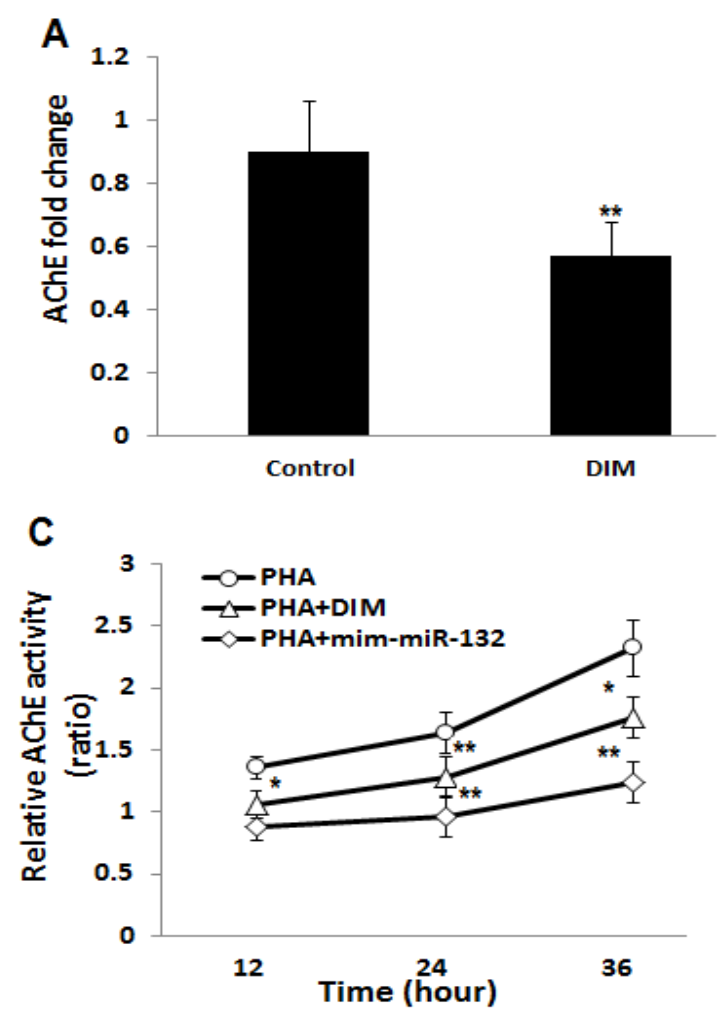

the results confirm the hypothesis that DIM induces miR-132/AChE module and potentiates cholinergic anti-inflammation comparable to that induced by TCDD.

B
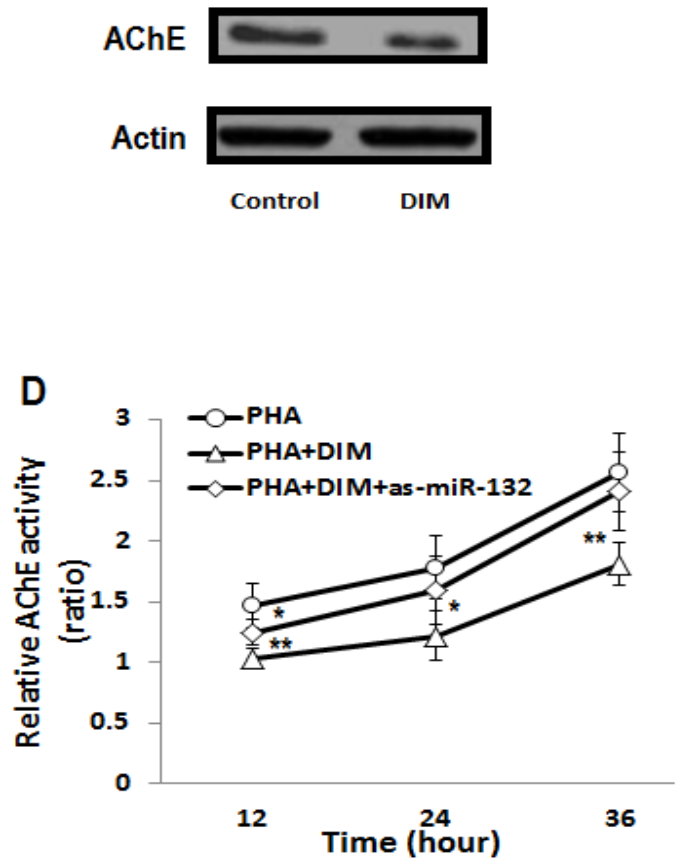

Figure 2. DIM induces miR-132/AChE module and potentiates cholinergic anti-inflammation. (A and B) gene expression of $\mathrm{AChE}$ in spleens of control and EAE mice 10 days after immunization. (C, D) Catalytic activity of $\mathrm{AChE}$ in $\mathrm{CD}^{+}$cells culture stimulated with PHA. The mim-miR-132 was transfected in PHA-treated cells, as-miR132 was transfected in PHA+DIM-treated cells, other treatments were transfected with non-specific nucleotides. ${ }^{*} p<0.05, * * p<0.01$. Data are shown as mean $\pm \mathrm{SD}$ of pooled data from three independent experiments. (A) $\mathrm{n}=5$, (B and C) $n=5$.

\section{DIM-induced cholinergic anti- inflammation is characterized by decreased proliferation of $\mathrm{CD4}^{+}$cell}

To describe the cholinergic antiinflammation induced by DIM in EAE mice, the proliferation of spleen $\mathrm{CD}^{+}$cells was studied. The DIM treatment suppressed the proliferative response of $\mathrm{CD}^{+}$cells from EAE mice to $\mathrm{MOG}_{35-5}$ restimulated (Fig. $3 \mathrm{~A})$. To investigate a direct role of miR-132 in cell proliferation, the cells were transfected with mim-miR-132. An over- expression of miR-132 exerted inhibitory effects on cell proliferation (Fig. 3B). To confirm the inhibitory effect of DIM on $\mathrm{CD}^{+}$cell proliferation, miR-132 was inhibited by as-miR-132 transfection. In line with this observation, knockdown of miR132 in $\mathrm{CD}^{+}$cell from DIM-treated EAE mice partially mitigated the inhibitory effect of DIM (Fig. 3C). These results suggest that miR-132/AChE module is implicated in the suppressive effects of DIM on $\mathrm{CD}^{+}{ }^{+} \mathrm{T}$-cells. 


\section{IL-17 and IFN- $y$ are decreased as a component of the DIM-induced cholinergic anti-inflammation}

To further characterize the cholinergic anti-inflammatory system induced by DIM, production of proinflammatory and anti-inflammatory cytokines in $\mathrm{CD}^{+}$cells was measured in response to restimulation with $\mathrm{MOG}_{35-55}$. As shown in Fig. 3D, DIM suppressed IL-17 and IFN- $\gamma$. The mim-miR-132 suppressed IL-17 and IFN- $\gamma$ to levels comparable to those of the DIM treatment. A slight enhancing effect was observed on TGF- $\beta$ with DIM, while no significant effect was observed on IL-10 (Fig. 3E). The results here show that the cholinergic antiinflammatory system augmented by DIM was characterized by decreased levels of IL17 and IFN- $\gamma$.
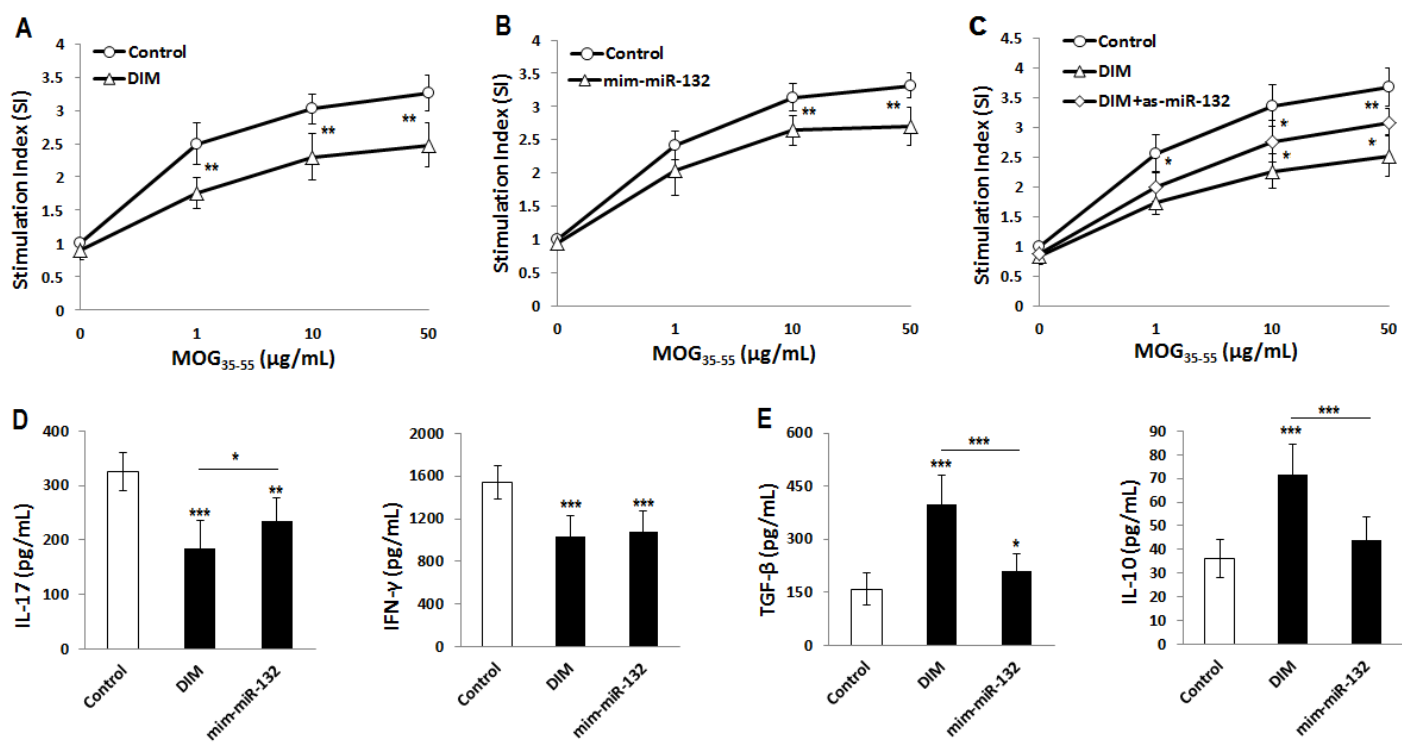

Figure 3: Suppressed proliferation and pro-inflammatory cytokines in $\mathrm{CD}^{+}$cells are components of the DIMinduced cholinergic anti-inflammatory system. (A) Proliferative response of $\mathrm{CD}^{+}{ }^{+}$cells to restimulation with $\mathrm{MOG}_{35-55}$ for 3 days. (B) Proliferative response of $\mathrm{CD}^{+}$cells transfected with mim-miR-132 or non-specific nucleotides and restimulated with $\mathrm{MOG}_{35-55}$ for 3 days. ${ }^{*} p<0.01$. (C) Proliferative response of $\mathrm{CD}^{+}$cells from DIM-treated mice transfected with as-miR-132 or non-specific nucleotides and restimulated with $\mathrm{MOG}_{35-55}$ for 3 days. Proliferative response is shown as stimulation index $(\mathrm{SI})=$ proliferation of unstimulated cells/proliferation of $\mathrm{MOG}_{35-55}$-treated cells. (D \& E) Cytokines level in culture supernatant of $\mathrm{CD}^{+}$cells from EAE mice and restimulated with $\mathrm{MOG}_{35-55}$ for 3 days. ${ }^{*} p<0.05$, $* * p<0.01, * * * p<0.001$. Data are shown as mean \pm SD of pooled data from three independent experiments. (A-E) $n=5$.

\section{DISCUSSION}

The MS is an autoimmune disorder driven mainly by $\mathrm{T}$ cell to target the myelin sheath of the central nervous system (CNS). Important advances have been achieved to understand the pathogenesis of this disease, and different therapeutic strategies have been proposed (Di Mitri et al 2015; Izquierdo et al 2015; Schmitt 2015). However, full understanding of the disease development or effective treatments is not yet established.

Due to the similarities with the inflammatory features of MS, the EAE mouse model is considered as one of the best available models to study the MS. An increasing paradigm has shown that $\mathrm{Ahr}$ modulates different inflammatory parameters in EAE (Quintana et al 2008; Durate et al 2013). Importantly, activation of Ahr by exogenous agonists such as DIM and 
TCDD attenuates the EAE inflammatory symptoms (Quintana et al 2008; Rouse et al 2013). In parallel, recent studies have added a new dimension to understand the development of MS by linking its development to many miRNAs (Noorbakhsh et al 2011; Lewkowicz et al 2015), and several studies have shown that modulation of certain miRNAs exhibits therapeutic properties in EAE (Du et al 2009; O'Connell et al 2010; Guan et al 2013; Liu et al 2014). Nevertheless, the axis of Ahr with the relevant miRNAs is rarely investigated.

The Current study, for the first time, shows that DIM alleviates EAE symptoms by inducing miR-132/AChE module, which potentiates cholinergic anti-inflammatory system to attenuate EAE. This antiinflammatory system is characterized by suppression of cell proliferation and production of the pro-inflammatory cytokines IL-17 and IFN- $\gamma$ in the encephalitogenic $\mathrm{CD}^{+}$cells. The DIMinduced cholinergic anti-inflammation was confirmed by over-expression or silencing miR-132. Since the MS is driven mainly by the $\mathrm{CD}^{+}$cells, current study focused on this $\mathrm{T}$ cell subpopulation.

Although miR-132 along with miR212 constitute a cluster named miR-212/132, miR-132 only is highly expressed and possesses demonstrated function in the CNS such as neuronal differentiation and cognitive processes (Remenyi et al 2010; Tognini et al 2011; Haenisch et al 2015). Previously, we found that the highly toxic Ahr against TCDD induces miR-132/AChE module and potentiates cholinergic antiinflammation in EAE mice (Hanieh and Alzahrani 2013). The current work examined the association of the natural Ahr agonist DIM with the miR-132/AChE module in EAE mice. By gain and loss of function approach, the results approved that DIM has a regulatory function on the miR132/AChE module in vitro and in vivo, suggesting safe application of this Ahr agonist. Consistently, independent studies demonstrated that miR-132 potentiates cholinergic anti-inflammation by targeting AChE mRNA in response to LPS treatment (Shaked et al 2009; Liu et al 2015).

It is reported here that DIM induces miR-132/AChE module suppresses proliferation of the encephalitogenic $\mathrm{CD} 4^{+}$ cells. Also, it is found that attenuating the cholinergic anti-inflammation by silencing miR-132 in $\mathrm{CD}^{+}$cells restores proinflammatory cytokines production and cell proliferation capacities. In line, the $\mathrm{AChE}$ inhibitors such as EN101 and huperzine A augment cholinergic anti-inflammatory system (Nizri et al 2006; Wang et al 2012). This system includes a reduced production of pro-inflammatory cytokines and proliferative response of T-cell.

It has been demonstrated that antagonizing the effects of AChE by stimulation of $\alpha 7$ nicotinic receptor suppresses the production of the IL-1 $\beta$ and TNF- $\alpha$ in macrophages (Yoshikawa et al 2003). Also, inhibition of AChE activity decreases the production of IL-17 and IFN- $\gamma$, and TNF- $\alpha$ in T cells (Nizri et al 2006; Wang et al 2012). Consistently, the results here provides evidence that DIM alleviates EAE by reducing levels of AChE, IL-17, IFN- $\gamma$, IL-1 $\beta$, IL-6 and TNF- $\alpha$. These observations support the findings of the current study that the decreased proinflammatory cytokines by DIM treatment is part of the induced cholinergic antiinflammatory system. Alternatively, miR132 might have directly decreased IL-6 and IL-1 $\beta$ by targeting their miRNAs (Lagos et al 2010). Taken together, it is likely that other immune cell compartments are involved in mediating the anti-inflammatory properties of DIM, which warrants more investigation. 
In summary, current study provides the first evidences that the non-toxic Ahr agonist DIM regulates the expression of miR-132 in $\mathrm{CD}^{+}$cells. Also, it identifies a new mechanism through which DIM induces miR-132 and potentiates cholinergic antiinflammatory system in the mouse model of MS. The results pave intriguing possibilities for using miR-132 mimics and/or DIM to control autoimmune inflammation in MS patients.

\section{ACKNOWLEDGMENT}

This study was funded by the Deanship of Scientific Research, King Faisal University (Grants 15008 and 160030).

Conflict of interest: The author declares no financial conflict of interest.

\section{REFERENCES}

Boldin M.P., Taganov K.D., Rao D.S., Yang L., Zhao J.L., Kalwani M., GarciaFlores Y. et al. (2011). miR-146a is a significant brake on autoimmunity, myeloproliferation, and cancer in mice. J. Exp. Med. 208:1189-1201.

Chinen I., Nakahama T., Kimura A., Nguyen N.T., Takemori H., Kumagai A., Kayama H. et al. (2015). The aryl hydrocarbon receptor/microRNA212/132 axis in T cells regulates IL-10 production to maintain intestinal homeostasis. Int. Immunol. 27(8):40515.

de Yébenes V.G., Bartolomé-Izquierdo N., Ramiro A.R. (2013). Regulation of Bcell development and function by microRNAs. Immunol. Rev. 253(1):2539.

Di Mitri D., Sambucci M., Loiarro M., De Bardi M., Volpe E., Cencioni M.T., Gasperini C. et al. (2015). The p38
MAPK cascade modulates TH17 differentiation and functionality in Multiple Sclerosis. Immunol. doi: 10.1111/imm.12497.

Dong L., Xia S., Gao F., Zhang D., Chen J., Zhang J. (2010). 3,3'-diindolylmethane attenuates experimental arthritis and osteoclastogenesis. Biochemical. Pharmacol. 79(5):715-721.

Du C., Liu C., Kang J., Zhao G., Ye Z., Huang S., Li Z. et al. (2009). MicroRNA miR-326 regulates TH-17 differentiation and is associated with the pathogenesis of multiple sclerosis. Nat. Immunol. 10:1252-1259.

Duarte J.H., Di Meglio P., Hirota K., Ahlfors H., Stockinger B. (2013). Differential influences of the aryl hydrocarbon receptor on Th17 mediated responses in vitro and in vivo. PLoS One. 14;8(11):e79819.

Ellman G.L., Courtney K.D., Andres V.Jr, Feather-stone R.M. (1961). A new and rapid colorimetric determination of acetylcholinesterase activity. Biochem. Pharmacol. 7:88-95.

Filková M., Aradi B., Senolt L., Ospelt C., Vettori S., Mann H., Filer A. et al. (2014). Association of circulating miR223 and miR-16 with disease activity in patients with early rheumatoid arthritis. Ann. Rheum. Dis. 73(10):1898-904.

Guan H., Fan D., Mrelashvili D., Hao H., Singh N.P., Singh U.P., Nagarkatti P.S. et al. (2013). MicroRNA let-7e is associated with the pathogenesis of experimental autoimmune encephalomyelitis. Eur. J. Immunol. 43:104-114.

Haenisch S., Zhao Y., Chhibber A., Kaiboriboon K., Do L.V., Vogelgesang 
S., Barbaro N.M. et al. (2015). SOX11 identified by target gene evaluation of miRNAs differentially expressed in focal and non-focal brain tissue of therapy-resistant epilepsy patients. Neurobiol. Dis. 77:127-40.

Hanieh H. (2014). Toward understanding the role of aryl hydrocarbon receptor in the immune system: current progress and future trends. Biomed. Res. Int. 2014:520763.

Hanieh H., Alzahrani A. (2013). MicroRNA132 suppresses autoimmune encephalomyelitis by inducing cholinergic anti-inflammation: a new Ahr-based exploration. Eur. J. Immunol. 43(10):2771-82.

Izquierdo G., García-Agua Soler N., Rus M., García-Ruiz A. J. (2015). Effectiveness of glatiramer acetate compared to other multiple sclerosis therapies. Brain Behav. 5(6):e00337.

Junker A., Krumbholz M., Eisele S., Mohan H., Augstein F., Bittner R., Lassmann H. et al. (2009). MicroRNA profiling of multiple sclerosis lesions identifies modulators of the regulatory protein CD47. Brain. 132:3342-3352.

Lagos D., Pollara G., Henderson S., Gratrix F., Fabani M., Milne R.S., Gotch F., Boshoff C. (2010). miR-132 regulates antiviral innate immunity through suppression of the p300 transcriptional co-activator. Nat. Cell. Biol. 12:513-519.

Lewkowicz P., Cwiklińska H., Mycko M.P., Cichalewska M., Domowicz M., Lewkowicz N., Jurewicz A., Selmaj K.W. (2015). Dysregulated RNAInduced Silencing Complex (RISC) Assembly within CNS Corresponds with Abnormal miRNA Expression during
Autoimmune Demyelination. $J$. Neurosci. 35(19):7521-37.

Liu G., Abraham E. (2013). MicroRNAs in immune response and macrophage polarization. Arterioscler. Thromb. Vasc. Biol. 33(2):170-7.

Liu F., Li Y., Jiang R., Nie C., Zeng Z., Zhao N., Huang C. et al. (2015). miR132 inhibits lipopolysaccharide-induced inflammation in alveolar macrophages by the cholinergic anti-inflammatory pathway. Exp. Lung. Res. 41(5):261-9.

Liu X., He F., Pang R., Zhao D., Qiu W., Shan K., Zhang J. et al. (2014). Interleukin-17 (IL-17)-induced microRNA 873 (miR-873) contributes to the pathogenesis of experimental autoimmune encephalomyelitis by targeting A20 ubiquitin-editing enzyme. J. Biol. Chem. 289(42):28971-86.

Masuda K., Kimura A., Hanieh H., Nguyen N.T., Nakahama T., Chinen I., Otoyo Y. et al. (2011). Aryl hydrocarbon receptor negatively regulates LPS-induced IL-6 production through suppression of histamine production in macrophages. Int. Immunol. 23:637-645.

Murata K., Yoshitomi H., Tanida S., Ishikawa M., Nishitani K., Ito H., Nakamura T. (2010). Plasma and synovial fluid microRNAs as potential biomarkers of rheumatoid arthritis and osteoarthritis. Arthritis Res. Ther. 12:R86.

Murugaiyan G., da Cunha A.P., Ajay A.K., Joller N., Garo L.P., Kumaradevan S, Yosef N. et al. (2015). MicroRNA-21 promotes Th17 differentiation and mediates experimental autoimmune encephalomyelitis. J. Clin. Invest. 125(3):1069-80. 
Nakahama T., Hanieh H., Nguyen N.T., Chinen I., Ripley B., Millrine D., Lee S. et al. (2013). Aryl hydrocarbon receptormediated induction of the microRNA132/212 cluster promotes interleukin-17producing T-helper cell differentiation. Proc. Natl. Acad. Sci. USA. 110:119649.

Nakahama T., Kimura A., Nguyen N.T., Chinen I., Hanieh H., Nohara K., FujiiKuriyama Y. et al. (2011). Aryl hydrocarbon receptor deficiency in $\mathrm{T}$ cells suppresses the development of collagen-induced arthritis. Proc. Natl. Acad. Sci. USA. 108:14222-14227.

Nizri E., Hamra-Amitay Y., Sicsic C., Lavon I., Brenner T. (2006). Anti-inflammatory properties of cholinergic up-regulation: A new role for acetylcholinesterase inhibitors. Neuropharmacol. 50:540547.

Nizri E., Irony-Tur-Sinai M., Faranesh N., Lavon I., Lavi E., Weinstock M., Brenner T. (2008). Suppression of neuroinflammation and immunomodulation by the acetylcholinesterase inhibitor rivastigmine. J. Neuroimmunol. 203:1222.

Nguyen N.T., Hanieh H., Nakahama T., Kishimoto T. (2013). The roles of aryl hydrocarbon receptor in immune responses. Int. Immunol. 25(6):335-43.

Noorbakhsh F., Ellestad K.K., Maingat F., Warren K.G., Han M.H., Steinman L., Baker G.B., Power C. (2011). Impaired neurosteroid synthesis in multiple sclerosis. Brain. 134:2703-2721.

O'Connell R.M., Kahn D., Gibson W.S., Round J.L., Scholz R.L., Chaudhuri A.A., Kahn M.E. et al. (2010).
MicroRNA-155 promotes autoimmune inflammation by enhancing inflammatory $\mathrm{T}$ cell development. Immunity. 33:607-619.

Pauley K.M., Satoh M., Chan A.L., Bubb M.R., Reeves W.H. Chan E.K. (2008). Upregulated miR-146a expression in peripheral blood mononuclear cells from rheumatoid arthritis patients. Arthritis Res. Ther. 10:R101.

Quintana F.J., Basso A.S., Iglesias A.H., Korn T., Farez M.F., Bettelli E., Caccamo M.O. et al. (2008). Control of Treg and TH17 cell differentiation by the aryl hydrocarbon receptor. Nature. 453:65-71.

Rouse M., Singh N.P., Nagarkatti P.S., Nagarkatti M. (2013). Indoles mitigate the development of experimental autoimmune encephalomyelitis by induction of reciprocal differentiation of regulatory T cells and Th17 cells. Br. J. Pharmacol. 169(6):1305-21.

Schmitt N. (2015). Role of T Follicular Helper cells in Multiple Sclerosis. J. Nat. Sci. 1(7):e139.

Shaked I., Meerson A., Wolf Y., Avni R., Greenberg D., Gilboa-Geffen A. (2009). MicroRNA-132 potentiates cholinergic anti-inflammatory signalling by targeting acetylcholinesterase. Immunity. 31:965-973.

Singh N.P., Singh U.P., Singh B., Price R.L., Nagarkatti M., Nagarkatti P.S. (2011). Activation of aryl hydrocarbon receptor (AhR) leads to reciprocal epigenetic regulation of FoxP3 and IL-17 expression and amelioration of experimental colitis. PLoS One. 6(8):e23522. 
Stockinger B., Veldhoen M. and Hirota K. (2009). Modulation of Th17 development and function by activation of the aryl hydrocarbon receptor--the role of endogenous ligands. Eur. J. Immunol. 39:652-654.

Tognini P., Putignano E., Coatti A., Pizzorusso T. (2011). Experiencedependent expression of miR-132 regulates ocular dominance plasticity. Nat. Neurosci. 14:1237-9.

Wang J., Chen F., Zheng P., Deng W., Yuan J., Peng B., Wang R. et al. (2012). Huperzine A ameliorates experimental autoimmune encephalomyelitis via the suppression of $\mathrm{T}$ cell-mediated neuronal inflammation in mice. Exp. Neurol. 236:79-87.
Xiao C., Srinivasan L., Calado D.P., Patterson H.C., Zhang B., Wang J., Henderson J.M. et al. (2008). Lymphoproliferative disease and autoimmunity in mice with increased miR-17-92 expression in lymphocytes. Nat. Immunol. 9:405-414.

Yoshikawa H., Kurokawa M., Ozaki N., Nara K., Atou K., Takada E., Kamochi H., Suzuki N. (2003). Nicotinic acetylcholine receptor alpha7 subunit is an essential regulator of inflammation. Nature. 421:384-388.

Zhu S., Pan W., Qian Y. (2013). MicroRNA in immunity and autoimmunity. J. Mol. Med. (Berl). 91(9):1039-50.

\section{ثنائي اندول الميثين يزيد نسخ ميكرورنا-132 و يعزز نظام كوليني مضاد لإلتهاب المناعة الذاتية في مرض التصلب اللويحي المتعدد

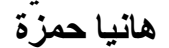

الميكرورنا (microRNA) هي مجموعة من النيكليوتيدات الر اييوزية التي لا نترجم لبروتينات، و تعمل على تنظيم ترجمة

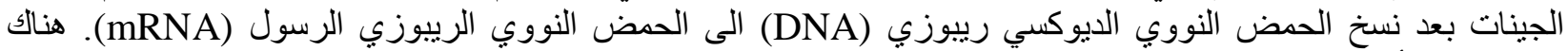

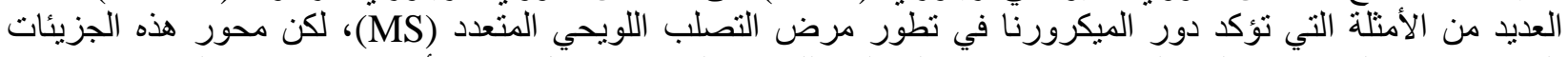

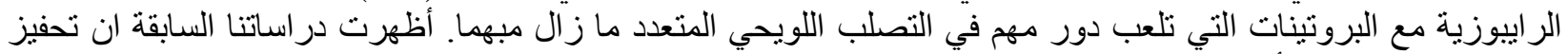

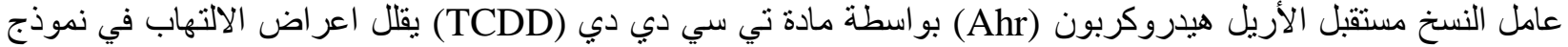

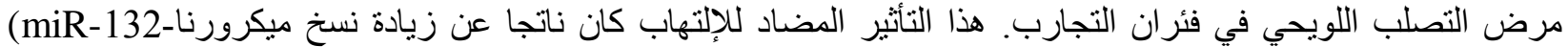

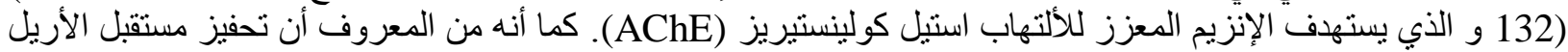

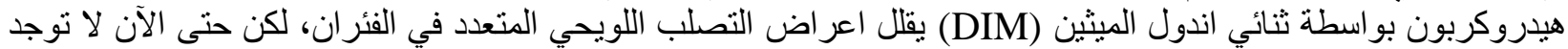
أبحاث قامت بدر اسة محور ثنائي اندول الميثين مع الميكرورنا.

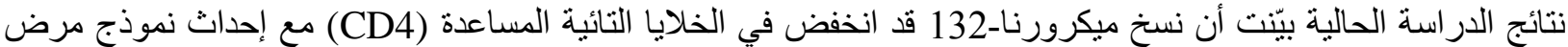

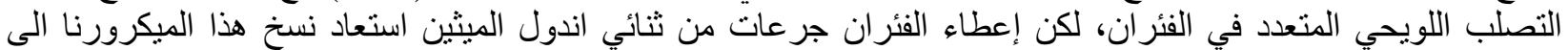

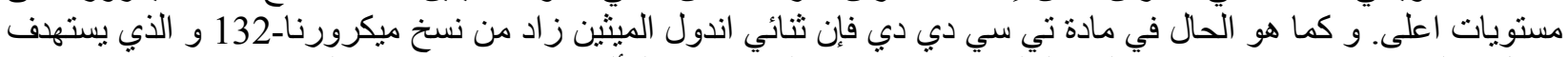

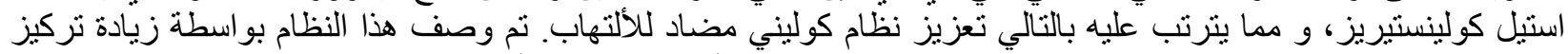

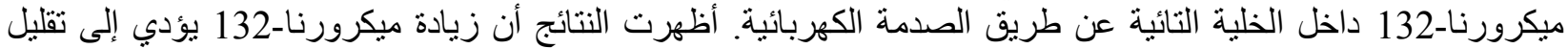

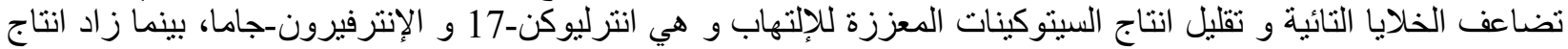

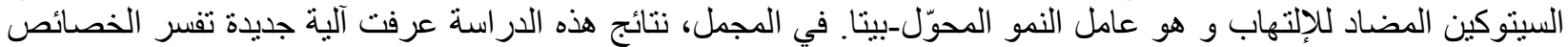

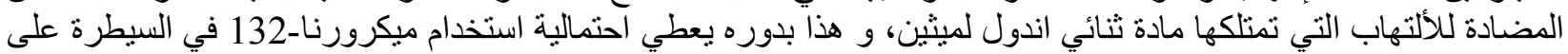
الإلتهابات المناعية الذاتية في مرض التصلي مادلب اللويحي المتعدد. 